\title{
A current perspective on cancer immune therapy: step-by-step approach to constructing the magic bullet
}

\author{
Gabriele D'Errico ${ }^{1}$, Heather L. Machado ${ }^{2^{*}}$ (1) and Bruno Sainz Jr. ${ }^{1,3,4^{*}}$
}

\begin{abstract}
Immunotherapy is the new trend in cancer treatment due to the selectivity, long lasting effects, and demonstrated improved overall survival and tolerance, when compared to patients treated with conventional chemotherapy. Despite these positive results, immunotherapy is still far from becoming the perfect magic bullet to fight cancer, largely due to the facts that immunotherapy is not effective in all patients nor in all cancer types. How and when will immunotherapy overcome these hurdles? In this review we take a step back to walk side by side with the pioneers of immunotherapy in order to understand what steps need to be taken today to make immunotherapy effective across all cancers. While early scientists, such as Coley, elicited an unselective but effective response against cancer, the search for selectivity pushed immunotherapy to the side in favor of drugs focused on targeting cancer cells. Fortunately, the modern era would revive the importance of the immune system in battling cancer by releasing the brakes or checkpoints (anti-CTLA-4 and anti-PD-1/PD-L1) that have been holding the immune system at bay. However, there are still many hurdles to overcome before immunotherapy becomes a universal cancer therapy. For example, we discuss how the redundant and complex nature of the immune system can impede tumor elimination by teeter tottering between different polarization states: one eliciting anti-cancer effects while the other promoting cancer growth and invasion. In addition, we highlight the incapacity of the immune system to choose between a fight or repair action with respect to tumor growth. Finally we combine these concepts to present a new way to think about the immune system and immune tolerance, by introducing two new metaphors, the "push the accelerator" and "repair the car" metaphors, to explain the current limitations associated with cancer immunotherapy.
\end{abstract}

Keywords: CTLA-4, PD-1, PD-L1, Immunotherapy, Coley's toxin

\section{Introduction}

Since war was declared on cancer in 1971, our arsenal of drugs against this enemy has steadily increased. The first class of drugs developed, conventional chemotherapy [1], provided significant benefits for the treatment and management of different cancers; however, conventional chemotherapy has two intrinsic defects: lack of selectivity [2] and long-term resistance [3]. The subsequent development of targeted therapies, including monoclonal

\footnotetext{
*Correspondence: hmachado@tulane.edu; bruno.sainz@uam.es

${ }^{1}$ Department of Biochemistry, School of Medicine, Autónoma University of Madrid, Calle del Arzobispo Morcillo 4, 28029 Madrid, Spain

2 Department of Biochemistry and Molecular Biology, Tulane University School of Medicine, 1430 Tulane Ave, \#8543, New Orleans, LA 70112, USA Full list of author information is available at the end of the article
}

antibody-based therapies, such as rituximab, overcame the lack of selectivity associated with conventional chemotherapy by targeting specific proteins involved in cancer cell stimulation, proliferation or apoptosis evasion [4]. While monoclonal antibody-based strategies have significantly increased in clinical practice over the past decade, mechanisms of acquired resistance remains a hurtle, likely due to genetic and epigenetic instability of cancer cells [5]. Thus, despite significant advances made in cancer treatment over the past 45 years, we are still far from developing therapies capable of effectively ablating cancer while avoiding adverse effects on healthy cells and loss of efficacy over time.

Starting with Coley's toxin, followed by Paul Ehrlich's hypothesis of tumor surveillance and contrasted by 
Burnet's immunological tolerance theory [6], the idea that the immune system can play a pro- and/or antitumor role has been recognized and debated for years. As the immune system is armed to protect against pathogens, it has been long postulated that immune cells should recognize tumor cells as foreign, and effectively eliminate them before spreading to distant organs. This concept, today referred to as cancer immunotherapy, has the potential to be the magic bullet that investigators have been desperately searching for since the early 1900 s [7].

The last century was a pendulum, swinging between big hope and deep disappointment in the immunotherapy field. Why has our view of immunotherapy shifted from promising to disappointing? Can immunotherapy be made more effective? In this review we look to the past to understand the current problems associated with immunotherapy. We present a new way to think about the immune system and immune tolerance. In addition to the standard "fuel the engine, release the brake" rules of immunotherapy, we introduce the "push the accelerator" and "repair the car" metaphors to explain part of the current limitations associated with cancer immunotherapy.

\section{Immunotherapy: a revolutionary view of cancer treatment}

Immunotherapy was born in 1890. Its father, William Coley [8], observed that a patient with an inoperable sarcoma that suffered a Streptococcus pyogenes infection twice obtained complete remission. Based on this observation, Coley treated approximately 1000 patients with inoperable cancers (specially sarcomas) with a mixture consisting of killed S. pyogenes and Serratia marcescens, achieving a complete remission in $10 \%$ of treated patients. Compared with actual 5-year survival rates for metastatic sarcoma (20\%) [9], the effects of Coley's toxin were promising and demonstrated that our immune system could effectively eradicate tumors with a low rate of adverse effects in a subset of patients. While the mechanism of action of Coley's toxin was unknown at the time, Coley's toxin essentially activated tumor-infiltrating leukocytes [heterogeneous populations of cells, including varying proportions of neutrophils, macrophages, $\mathrm{T}$ and $\mathrm{B}$ cells, and natural killer cells (NK)]. Following administration of Coley's toxin, dendritic cells (DCs), professional antigen presenting cells (APCs), initiate an immune response by presenting the captured bacterial antigen to naïve $\mathrm{CD}^{+}$or $\mathrm{CD}^{+} \mathrm{T}$ cells [10] in lymphoid tissues, which results in $\mathrm{T}$ cell priming [11] and inflammatory interleukin production [IL-1, IL-2, tumor necrosis factor alpha (TNF $\alpha$,) IL-12] [12, 13]. Clonal T cell expansion triggers a humoral immune response by activating B cells, or cellular immunity by activating Th1 effector cells or NK cells [14]. Thus, as tumors often display a high degree of leukocyte infiltration, it is reasonable that stimulation of tumor-infiltrating leukocytes can result in tumor cell targeting and elimination.

\section{The first gauntlet: release the brake}

In 1949, some years after Coley's first experiments, Macfarlane Burnet stated, "if in embryonic life expendable cells from a genetically distinct race are implanted and established, no antibody response should develop against the foreign cell antigen when the animal takes on independent existence" $[6,15]$. Peter Medawar would go one step further and propose that the immune system becomes tolerant to cancer cells due to the similarities that exist with normal healthy cells. In his early experiments, Medawar injected embryonic mouse donor cells into mice of a different strain, rendering the recipient mice tolerant to future grafts from the donor and not third-party strains [16]. These experiments set the initial groundwork for what would become the concept of acquired immunological tolerance. Even if the molecular mechanisms explaining Medawar and Burnet's observations would not be discovered until 25 years later, Medawar and Burnet had already thrown down the first gauntlet to modern immunotherapy [16].

Immunological tolerance is a fundamental process, the lack of which would result in numerous pathologies including autoimmune illnesses. Since Burnet and Medawar won the Nobel Prize for their pioneering work in this field more than 50 years ago, an enormous amount of progress has been made to better understand immune tolerance and the numerous redundant mechanisms involved in this biological process. In 1959, Joshua Lederberg published nine propositions on immunity and tolerance [17]. The sixth proposition stated that "the immature antibody-forming cell is hypersensitive to an antigen-antibody combination: it will be suppressed if it encounters the homologous antigen at this time", highlighting that each antibody-producing cell has a single specificity [17]. In 1978, Nossal and Pike experimentally demonstrated that bone marrow-derived cells cultured with an antigen became tolerant to the antigen in a time-dependent manner, with maximal tolerance being achieved only when the antigen was present continuously as the cultured bone marrow cells matured [18]. In the late 1980's, Kappler demonstrated central tolerance in mice and indicated that tolerance induction may occur in the thymus [19]. Goodnow later showed that B cells that reactive to "self" antigens are eliminated or silenced in order to avoid autoimmunity [20]. And finally, Le Douarin demonstrated that the thymus is not only important for central tolerance but it produces cells [i.e. regulatory $\mathrm{T}\left(\mathrm{T}_{\text {reg }}\right)$ cells] that strongly regulate effector cells, discovering a third dominant form of immune tolerance [21]. 
Taken together these results clearly indicated that selfimmune tolerance is maintained and regulated by multiple mechanisms, including similarity with self-antigens, regulatory immune cells, a suppressive versus activating cytokine balance and immune checkpoints. Thus, if this process is to be effectively manipulated in order to release the brake, we have to stimulate APCs with an antigen significantly different from "self" to recruit effector cells, balance cytokines to obtain a pro-inflammatory milieu, and finally, inhibit immune checkpoints to avoid tolerance.

\section{Hope dies last}

The idea of immune tolerance put forth by Medawar and Burnet dampened the hope generated by Coley's experiments that activating the immune system could treat cancer. However, in 1953, Foley demonstrated that methylcholanthrene-induced tumor cells could produce immunogenic antigens in mice, although strain-dependent differences were observed [22]. Nathrath would add additional fuel to the fire by showing that the host immune system is capable of recognizing new molecular properties (antigens) displayed by tumor cell as a result of the changes accumulating during the transition from normal to malignant cells [23]. Interestingly, however, the greatest hope for immunotherapy would come from another illness described in the 1950s, autoimmune diseases such as lupus, which clearly confirmed that auto-reactive cells can elude self tolerance [24]. Finally, towards the later end of the 20th century, immunogenic tumor associated antigens were discovered in mice [25] and humans [26], resulting from cancer cell genomic instability. Unsettling still was the fact that while cancer cells could express a multitude of new and unknown antigens as a result of malignant transformation, the immune system was not stimulated to target these antigens/cells. The problem therefore was no longer the absence of immunogenic antigens on cancer cells, but rather understanding why our immune system "ignores/tolerates" cells harboring these antigens.

In the absence of inflammation, naïve T-cells circulate preferentially to secondary lymphoid tissue [27]. During an infection, APCs respond to inflammatory cytokines (IL-1, TNF- $\alpha$ ) [28] and migrate via afferent lymph vessels to lymph nodes where they can interact with naïve T-cells. In a similar manner, macrophage or B cells can take up and process free antigen in the blood or spleen [29]. The interaction of T cell CD28 and DC CD80 (B7-1) or CD86 (B7-2) allows for specific T cells to proliferate in the paracortex [30] and become competent to receive further activation signals from antigen-bearing macrophages and B cells. This process results in the production of cytokines, loss of L-selectin (which is involved in lymph node entry) and increased expression of adhesion molecules like VLA 4 (which facilitates extravasation into non-lymphoid tissue) [31]. Macrophages and parenchymal cells produce inflammatory cytokines (IL-1, TNF- $\alpha$ ) that increase expression of selectins and integrin ligands. Ultimately, activated T-cells express adhesion molecules that allow them to selectively enter inflamed tissues expressing counterpart adhesion molecules. In summary, immune cells can present antigens (dendritic cells, macrophages), produce cytokines (macrophage) and interact with cells of the adaptive immune system (B-cells, T-cells) in the lymph nodes, resulting in their subsequent activation via receptor/ligand interactions. Once activated, adaptive immune cells proliferate, alter their receptors and adhesion molecules, and finally migrate to initiate the destruction of foreign pathogens.

To avoid indiscriminate activation and self-destruction, the immune system has developed redundant mechanisms to tolerate self or non-dangerous antigens. Since central tolerance is not the underlying mechanisms by which cancer cells escape immune targeting, we refer the reader to several published reviews detailing the biology of central tolerance (Ref. [32, 33]). Peripheral tolerance, on the other hand, is the primary mechanism utilized by cancer cells to avoid the immune system. In the early 1990s, Jenkins and Schwartz demonstrated that T-cells need a co-stimulatory signal to fully activate, and if T-cells receive only TCR signals they become anergic [34]. The co-stimulatory signals must be received from APCs [35]: B7-1 (CD80) or B7-2 (CD86) on APCs binding to CD28 on T-cells is necessary to fully stimulate T-cells. While an important step forward in our understanding of T-cell regulation, the big discovery was not B7-1 or B7-2, but rather the receptor that inhibits the second co-stimulatory signal. James Allison, director of the UC Berkeley Cancer Research Laboratory, was intrigued with a molecule called cytotoxic T-lymphocyte antigen-4 (CTLA-4) [36], originally discovered in a cDNA library derived from activated T-cells. In the late 1990s, Allison and his group began to study how CTLA-4 inhibits T-cells and if this inhibition could explain why T-cells do not attack cancer cells. They demonstrated that CTLA4 , a homologue of $\mathrm{CD} 28$, bound with higher affinity (at least 10-fold) to both B7-1 and B7-2 [37] and inhibited $\mathrm{CD}^{+}{ }^{+} \mathrm{T}$-cell activation. Under certain conditions, T-cells up-regulate CTLA-4, which binds to B7-1 and B7-2 with a higher affinity than CD28, effectively hijacking the second co-stimulatory signal that T-cells require for full T-cell activation, proliferation, and effector function [38]. As a result, T-cells cannot be fully activated and thus undergo anergy. Since its discovery, several groups have worked diligently towards dissecting the role of CTLA4. Studies with CTLA- $4^{-1-}$ mice confirmed its inhibitory 
function in vivo. Waterhouse et al. showed that mice lacking CTLA-4 died early on of fatal lymphoproliferative disorders [39], demonstrating that CTLA-4 acts as a negative regulator of $\mathrm{T}$ cell activation and is vital for the control of lymphocyte homeostasis. Based on ever increasing data demonstrating a role for CTLA-4 as a negative regulator of T-cell activation, Allison and colleagues went on to show that in vivo administration of antibodies to CTLA-4 promoted the rejection of tumors, including pre-established tumors, confirming that CTLA-4 blockage can allow for, and potentiate, an effective immune responses against tumors [40].

\section{The first victory in the new era}

These studies and others [41-43] suggested that monoclonal antibody-mediated CTLA-4 blockage could represent an effective anti-cancer therapy. To translate these findings to the clinical setting, the Medarex Corporation generated a series of monoclonal antibodies using a unique transgenic mouse (HuMAb), in which the endogenous murine immunoglobulin genes had been knocked out and replaced with the human loci [44]. Ipilimumab showed safety in a phase I study and efficacy in a phase III study (ClinicalTrials.gov Identifier: NCT00094653) with primary overall survival endpoints. Patients allocated to receive ipilimumab had a median overall survival of 10.1 versus 6.4 months for the control group [hazard ratio (HR), 0.68; $P \leq 0.003$ ]. Based on the promising results, Bristol-Meyer's Yervoy ${ }^{\circledR}$ (ipilimumab) was approved by the US Food and Drug Administration (FDA) and the European Medicines Agency (EMA) in 2011 for the treatment of metastatic melanoma [38].

While CTLA-4 was the first "checkpoint" inhibitor identified, the list of checkpoint immunomodulators continues to grow, with inhibitors of the programmed cell death protein 1/ligand pathway leading the way. Programmed cell death protein 1, also known as PD-1 and CD279 (cluster of differentiation 279) is a member of the CD28 superfamily expressed on activated CD4 ${ }^{+}$ and $\mathrm{CD}^{+}{ }^{+}$T-cells as well as NK and B-cells while programmed death-ligand 1 (PD-L1), also known as cluster of differentiation 274 (CD274) or B7 homolog 1 (B7$\mathrm{H} 1$ ), is expressed predominantly on APCs. The main role of PD-1 is to act like a stopwatch to limit the activity of T-cells in the "battle field" during the effector phase of T-cell activation in peripheral tissues and the tumor microenvironment via the delivery of negative signals upon interaction with its two ligands (PD-L1 or PD-L2). PD-L1 and PD-L2 compete for PD-1 [45] and upon binding both inhibit $\mathrm{T}$ cell proliferation, cytokine production and cell adhesion [46], although some contradictory data have suggested a costimulatory function [47]. T-cells begin to express PD-1 when activated and its expression increase over time $[48,49]$, while PD-L1 is expressed on APCs present in the inflamed tissue. PD- 1 is also highly expressed on $\mathrm{T}_{\text {reg }}$ cells, where it may enhance their proliferation in the presence of ligand [50]. The expression on $\mathrm{T}_{\text {reg }}$ cells also highlights the role PD-1 plays in regulating the induction and maintenance of peripheral tolerance and protection from autoimmune attack [51]. Our current understanding of the PD-1/PD-L1 pathway shows that engagement of PD-1 and PD-L1 leads to inactivation of effector T-cell molecules such as Zap70 to inhibit T-cell proliferation, thus limiting the inflammatory damage in inflamed tissues [52]. For example, during chronic infections the PD-1/PD-L1 pathway leads to anergy [53]. It appears as though several cancers, including lung, ovarian and colon carcinomas as well as melanomas, have evolved to over express PD-L1 [54]. Thus, chronic tumor associated antigen exposure in cancer can lead to high levels of PD-1 expression on T-cells, which can interact with PD-L1 expressed on cancer cells, inhibiting T-cell activation and perhaps inducing a state of anergy in immune cells present in the tumor. Blocking the PD-1/PD-L1 pathway can revert this condition, promoting cancer cell elimination by activated T-cells. In the past 5 years, Bristol-Myers Squibb has produced a fully humanized antibody against PD-1 named Opdivo (Nivolumab), which obtained FDA accelerated approval in 2014 [55] based on the "Study of Nivolumab (BMS936558) Compared With Dacarbazine in Untreated, Unresectable, or Metastatic Melanoma".

Today many check-point inhibitors are used by oncologist to achieve significant increases in survival rates (e.g. 1 - and 2-year survival rates of 62 and $43 \%$, respectively for melanoma or 1- and 2-year survival rates of 42 and $23 \%$, respectively for lung cancer) and/or to achieve a durable partial or complete response in cancer patients (e.g. 31\% for melanoma patients) [56, 57]. Table 1 summarizes the current FDA- and EMA-approved immune checkpoint inhibitors. Clinical studies have also investigated the efficacy of combination therapies using antiPD-1/PD-L1 therapies together with other checkpoint inhibitors, such as the anti-CTLA4 treatment ipilimumab. The combination of nivolumab and ipilimumab increased overall survival in patients with untreated melanoma. The median progression-free survival was 11.5 months (95\% confidence interval [CI], 8.9-16.7) with nivolumab plus ipilimumab, as compared with 2.9 months (95\% CI, 2.8-3.4) with ipilimumab (hazard ratio for death or disease progression, $0.42 ; 99.5 \% \mathrm{CI}$, $0.31-0.57 ; \mathrm{P}<0.001)$, and 6.9 months (95\% CI, 4.3-9.5) with nivolumab (hazard ratio for the comparison with ipilimumab, 0.57; 99.5\% CI, 0.43-0.76; P < 0.001) [58]. It is important to stress that even if combination therapy allows us to obtain better results and improved medium 
Table 1 Summary of immune therapies in clinical use

\begin{tabular}{|c|c|c|c|c|}
\hline Immune therapy & Target & Stage & Cancer type & Ref. \\
\hline Ipilimumab & CTLA-4 & Clinical use & Advanced melanoma & 107 \\
\hline Nivolumab & PD-1 & Clinical use & $\begin{array}{l}\text { Melanoma } \\
\text { Renal cancer } \\
\text { NSLC }\end{array}$ & $\begin{array}{l}108 \\
109 \\
110\end{array}$ \\
\hline Pembrolizumab & PD-1 & Clinical use & Melanoma & 111 \\
\hline Atezolizumab & PD-L1 & Clinical use & $\begin{array}{l}\text { NSLC } \\
\text { Clear renal cancer bladder cancer }\end{array}$ & $\begin{array}{l}112 \\
113\end{array}$ \\
\hline Sipileucel & $\begin{array}{l}\text { Peripheral blood } \\
\text { mononuclear cells }\end{array}$ & Clinical use & Prostate cancer & 114 \\
\hline
\end{tabular}

overall survival, it is still far from becoming the perfect therapy. The current underlying problem with immune checkpoint inhibitors is that if there are no T-cells in the tumor border then there are no effector cells capable of eliminating the tumor cells. Therefore, even if we release the brake we cannot obtain clinically relevant results. Thus, the next strategy lies in "fueling the engine", that is allowing T-cells to reach the tumor border.

\section{Fueling the engine}

As stated above, immune checkpoints inhibitors are only effective if tumors are infiltrated with T-cells. Therefore, if a tumor lacks infiltrated T-cells, immune checkpoint inhibitors are essentially ineffective. Cancer vaccines have been extensively investigated as a strategy to induce T-cell infiltration in the tumor-"fuel the engine". Currently, however, Sipileucel is the only "cellular immunotherapy" (i.e. vaccine therapy) approved by the FDA. Sipileucel consists of autologous peripheral blood mononuclear cells (PBMCs), obtained by leukapheresis and cultured with a prostatic acid phosphatase linked to granulocyte-macrophage colony-stimulating factor (GM-CSF) [59]. Results from the 9902B study in patients with prostate cancer demonstrated an overall survival of 25.8 months for patients receiving Sipileucel compared to 21.7 months for patients who received the control treatment [59].

While Sipileucel demonstrates the clear benefit of "fueling the engine", the use of cancer vaccines in other tumor types is still in the experimental stages. For example, Kleponis et al. developed a GM-CSF-secreting pancreatic cancer vaccine (GVAX) that provided maturation signals to APCs at the local vaccine site. Stimulated APCs processed tumor antigens and presented them to T-effector cells, allowing T-cells to infiltrate the tumor [60]. The authors went on to show that PD-L1 expression was induced in the infiltrating cells (T-cells). This study demonstrated that vaccine-based therapies may have adequately primed pancreatic cancer for anti-PD-1/PD-L1 treatments, highlighting that this typically $\mathrm{T}$-cell-effector poor $/ \mathrm{T}_{\text {reg }}$-rich tumor [61] could be potentially treated with PD-1/PD-L1 inhibitors. Thus, cancer vaccine-based immunotherapy may overcome the resistance of certain cancers to immune checkpoint inhibitors, while immune checkpoint inhibitors may enhance the efficacy of the cancer-vaccine therapies. The goal of a combination strategy is to combine the strength of each immunotherapy approach, with cancer vaccines functioning to "fuel the engine" and immune checkpoint inhibitors working to "release the brake".

Perplexing is the fact that PD- 1 and CTLA- 4 checkpoint inhibitors, even when helped by cancer vaccines, are not effective against all cancer types, nor do they work in every patient with the same cancer. Perhaps other immune cell types are negatively affecting cancer immunotherapy? The explanation we put forward to explain this dilemma is that in some cancers we have the machinery (the car) on a downhill slope, so if we release the brake (immune checkpoint inhibitors) the car can move. In contrast, when we are on an uphill slope or on a plain field, releasing the brake simply does not move the car. For such scenarios, we have to release the brake and push the accelerator.

Looking deep inside the immune system we can find a dynamic and complex environment of cells that are different in type, size, complexity, markers and function. Even the same cell can exist in two (or more) different polarized states. For example, macrophages can switch between a pro-inflammatory (classically activated) and a reparative (alternatively activated) state [62]. T-cells can be stimulated into $\mathrm{T}$ effector cells or $\mathrm{T}_{\text {regs }}$ cells, each of which can have a very distinct role within a tumor [63]. Thus, we need to take a step back and understand why, when and how a cell (i.e. macrophage, T-cell, etc.) can switch from a classically activated (inflammatory macrophage or $\mathrm{T}$ effector cell) to an alternatively activated (pro-tumorigenic macrophage or $\mathrm{T}_{\text {reg }}$ ) state and what mediates this change. Answering these questions may provide the key to push the accelerator. 
Immune cells: Dr. Jekyll and Mr. Hide

The immune system is comprised of many cells, including but not limited to dendritic cells, mast cells, macrophages, neutrophils and lymphocytes. In addition, and to complicate the matter even more, immune cells are extremely plastic and each cell type can differentiate into at least two forms depending on their environment and the paracrine signals they receive (reviewed in [64-66]). Lymphocytes can differentiate into many subsets. Apart from $\mathrm{B}$ and $\mathrm{T}$ cells, $\mathrm{T}$ lymphocytes can differentiate into $\mathrm{CD}^{+}$or $\mathrm{CD}_{4}^{+} \mathrm{T}$ cells, the latter of which can in turn differentiate into $\mathrm{T}_{\text {reg }}$ and $\mathrm{T}_{\text {helper }}$ cells [67]. DCs that present captured antigens to naïve T-cells, have two major subsets: myeloid (i.e. conventional DC or immunogenic DC) and plasmacytoid form (or tolerogenic DC) [68]. As described above, macrophages can exist in at least two forms [69], and in the context of a tumor, macrophages and neutrophils can differentiate into tumor-associated macrophages (TAMs) or neutrophils (TANs) [70], respectively.

The interest in the different states of immune cells, particularly within the tumor, stems from the different biological affects these cells produce depending on their state, polarization or differentiation (Fig. 1). For example, human DCs can exist as immunogenic or tolerogenic DCs [71], with immunogenic DCs functioning primarily to stimulate a $\mathrm{T}_{\text {helper }}$ [72] response while tolerogenic DCs function primarily to stimulate a $\mathrm{T}_{\text {reg }}$ response [73]. Moreover, the ratio of different DCs depends on the cytokine milieu. In vitro studies clearly demonstrate that GM-CSF, interferon alpha (IFN $\alpha$ ), or IL-15 can induce the differentiation of inflammatory DCs while IL-10, vitamin A or D3, or immunosuppressive drugs such as cyclosporine A induce tolerogenic DCs through E-cadherin mediated signaling $[74,75]$. Thus, depending on the cytokine milieu, DCs can elicit a strong immune response or a tolerogenic state.

Macrophages represent another important cell type that play a pivotal role in activating and shaping the immune response, and similar to DCs, a dichotomy has been proposed for macrophage activation: classically or alternatively activated [76, 77]. IFN $\alpha$, LPS or inflammatory cytokines can induce classical activation while IL-4, IL-13, TGF- $\beta$ and reparatory signals can induce alternative activation of macrophages. In the last decade, if macrophages have gone from a negligible player in tumor progression to a pivotal modulator of tumor growth and metastasis, it is due to the identification of TAMs. There is now solid and continuously growing evidence to show that TAMs actively promote all aspects of tumor growth and development including promotion of angiogenesis, matrix remodeling and suppression of adaptive immunity [78]. Recent studies also show that TAMs share many characteristics with alternatively activated macrophages such as (1) activation of the arginase pathway, implicated in arginine metabolism; (2) promotion of cell repair and proliferation in strong opposition with the NOS pathway that promote cells killing [79]; (3) production of IL-10 and vascular endothelial growth factor (VEGF) over other factors promoting cell survival [80]; (4) production of matrix metalloproteinases (MMPs), implicated in cancer initiation and metastasis [81, 82]; (5) activation of NFKB and STAT3 signaling, enhancing tumor progression by directly communicating with cancer stem cells (CSCs) [62], and (6) activation of STAT6, which possess potent inhibitory activity of T-cell activity [83].

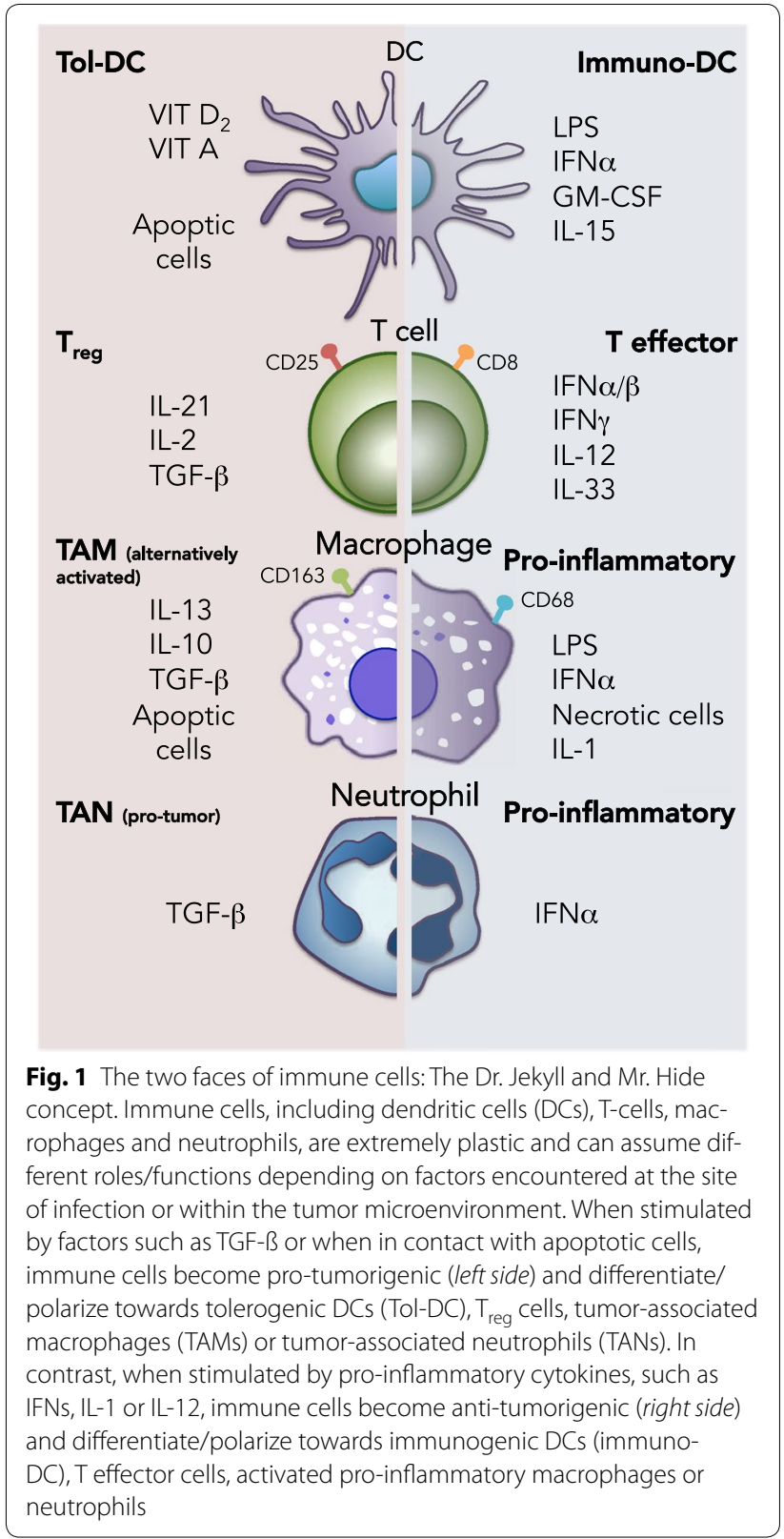


T-cells, the soldiers of the immune system, have a fundamental role in immune surveillance, and even for these cells a dichotomy exists: a T-cell can defend the host from cancer while aiding tumor growth. Zhang et al. showed that ovarian carcinoma patients with high tumor infiltrating lymphocytes had improved 5-year survival rates compared to patients with low tumor infiltrating lymphocytes [84]; however, in other cancers, such as renal cancer, high tumor infiltrating lymphocytes translated into worse prognosis [85]. These clear opposing observations are now explained by differences in the type of infiltrating T-cells: $\mathrm{T}_{\text {reg }}$ versus $\mathrm{T}$ effectors cells $\left(\mathrm{TCD} 8^{+}\right) . \mathrm{T}_{\text {reg }}$ cells, a subgroup of T-cells expressing CD4 and CD25, regulate activation of other T-cells and are necessary to maintain peripheral tolerance to self-antigens. We have now come to understand that increased numbers of $\mathrm{T}_{\text {reg }}$ cells present in the tumor can have a negative prognostic impact. For example, Sato et al. showed that a high $\mathrm{TCD}^{+} /$ $\mathrm{T}_{\text {reg }}$ cell ratio translated into better overall survival while the opposite was seen for a high $\mathrm{T}_{\text {reg }} / \mathrm{TCD} 8^{+}$ ratio [86]. Likewise, $\mathrm{Li}$ et al. demonstrated that the efficiency and percent depletion of $\mathrm{T}_{\text {reg }}$ cells from a tumor can improve cancer outcome [67, 87]. Specifically, they show that in Foxp3.LuciDTR-4 mice, which show 90-95\% $\mathrm{T}_{\text {reg }}$ depletion, large established tumors completely regressed, unlike anti-CD25 antibody-mediated $\mathrm{T}_{\text {reg }}$ elimination, which is less efficient (approximately $70 \%)$. Thus, high-level depletion of $\mathrm{T}_{\text {reg }}$ cells is necessary for tumor regression.

Like a coin, immune cells have two faces, one with a strong potential to fight cancer and the other (the opposite one) strongly promoting cancer development and immune system escape. This Dr. Jekyll and Mr. Hide concept has been re-coined the "corrupted policemen" concept by Bonavita et al. in order to stress the fact that those cells born to protect the host can become corrupt and turn against it to favor cancer growth [88]. This switch is complex and partly mediated by cytokines: inflammatory cytokines promoting immune system cells to show their anti-cancer face while anti-inflammatory cytokines promote the pro-cancer side to dominate. But cytokines are only mediators. More important are the stimuli that induce immune host cells to convert into either cancer allies (pro-tumor macrophages, $\mathrm{T}_{\text {reg }}$ cells, or tolerogenic DC) or cancer enemies (inflammatory macrophage, $\mathrm{CD}^{+}{ }^{+}$T-cells, $\mathrm{T}_{\text {helper }}$ cells or immunogenic DC) (Fig. 1). If we discover which mechanism(s) induce the inflammatory anti-cancer response and which ones induce the pro-cancer response, in theory we could pharmacologically change the face of the coin, favoring an anti-cancer response.

\section{To fight or to repair? That is the question}

The first problem that oncoimmunotherapists faced was immune tolerance. Currently, a more challenging dilemma lies in the ability of the immune system to balance itself between two opposing actions: "fight" the enemy or "repair the damage". A successful immune response can be accompanied by extensive tissue damage [89]. Fortunately, the immune system has the capacity to repair the resulting damage via $T_{\text {reg }}$ cells, macrophages and anti-inflammatory cytokines that send a repair message to the site of damage. In the context of cancer, however, the wrong choice can have detrimental effects on tumor eradication, as alluded to above. Numerous studies have shown that tumor growth and even post treatment tumor cell death can be perceived by the immune system as a repair signal, initiating a wound healing response that can favor sustained tumor growth and even tumor chemoresistance via immune cell secreted factors (reviewed in $[90,91]$ ). Dissecting how the immune system senses and responds to damage will improve our efforts of inhibiting the immune system from favoring tumor growth over tumor destruction.

After immune system activation, the battlefield is replete with damaged cells, the majority being apoptotic cells (e.g. bacteria, neutrophils, epithelial cells). It is therefore logical to think that these cells could regulate the immune system or represent the signal that promotes a repair response. Although conventional and targeted therapies often aim to induce apoptosis, these strategies may themselves be carcinogenic [92].

The relation between cancer, immune cells and dead/ apoptotic cells has gained increased attention over the past decade. Apoptosis obtained through activation of caspases or mitochondrial chain dysfunction was historically declared as non-immunogenic, while necrotic death accompanied by the release of proteins, lipids and other cellular debris from cells has been widely considered as strongly immunogenic $[93,94]$. Chemotherapy-mediated cell death was accepted as an apoptosis-mediated process, inducing an immunosuppressive milieu of cytokines [95], but studies show that depending on the agent used and the degree of cell death induced, chemotherapy can also induce necrotic cells [96]. Moreover, other studies have shown that classically-induced apoptotic cells can profoundly affect the immune system [97], and the idea that chemotherapy-induced apoptosis is non-immunogenic may be overstated [98-100].

In cancer, there appears to be a ying-yang scenario with respect to the presence of dead cells and method of cell death induction. The presence of apoptotic cells can be sensed and translated into a "need to repair" action, allowing $\mathrm{T}_{\mathrm{reg}} / \mathrm{Th} 2$ stimulation through cytokine 
expression (IL-10, IL-13) [100, 101]. On the other hand, necrotic cells or highly variable tumor associated antigens can be sensed and translated into a "need to fight" action, allowing $\mathrm{T}-\mathrm{CD} 4^{+}$stimulation thought inflammatory cytokine expression (TNF- $\alpha$, IL-1, etc.) [93]. Williams et al. showed that DCs exposed to apoptotic Jurkat cells or apoptotic primary T-cells failed to maturate and were unable to support $\mathrm{CD} 4^{+}$allogeneic $\mathrm{T}$-cell proliferation, as compared to DCs exposed to lipopolysaccharide (LPS) or necrotic cells [102]. Conversely, phosphatidylserine exposed on apoptotic epithelial cells suppressed IFN- $\beta$ production by dendritic cells via inhibitory signalling mediated by the cell-surface glycoprotein CD300a and thus suppressed $\mathrm{T}_{\text {reg }}$ cell proliferation [103]. Moreover, Kleinclauss et al. in 2006 demonstrated that apoptotic cells induce $\mathrm{CD} 4^{+} \mathrm{T}$-cells to express $\mathrm{CD} 25^{+}$(a marker of $\mathrm{T}_{\text {reg }}$ cells) inducing a state of tolerance [104]. Taken together, these studies demonstrate that the immune system can sense the type of danger and activate a specific action. In our laboratory we have discovered that apoptotic pancreatic tumor cells, as opposed to necrotic or live cells, can strongly induce immune system suppression or a pro-Th2 state, promoting repair signals that favor pancreatic tumor growth and chemoresistance (unpublished data). Our observations are in line with data published by Wu et al. where they show that intravenous administration of donor apoptotic splenocytes promotes the generation of tolerogenic DCs and the expansion of $\mathrm{T}_{\text {reg }}$ cells in the pancreas; in vivo clearance of either DCs or $\mathrm{T}_{\text {reg }}$ cells abrogated immune tolerance induction [105]. Thus, in pancreatic cancer where chemotherapies such as gemcitabine induce tumor cell apoptosis, an immune response that favors tumor growth may be activated.

\section{Conclusion}

The field of cancer immunotherapy has been driven by scientists such as William Coley whose important results set the foundation for modern day immunotherapy, as well as by scientists who while opposed the concept of immunotherapy made pinnacle discoveries that favored the evolution of immunotherapy into one of the most promising techniques in place today to battle cancer. The scientific community is convinced that immunotherapy is only a step away from becoming the magic bullet to defeat cancer. While the future looks promising, there are still many hurdles that need to be overcome.

We now have the capacity to "fuel the engine" by using vaccine strategies to accumulate effector T-cells at the tumor border, and "release the brake" to allow T-cells to fight the cancer by inhibiting immune system checkpoints. This strategy allows us to fight cancer when the battlefield is downhill or when the tumor entity itself is "immunogenic" due to (1) profound differences in tumor associated antigens between normal cells and tumor cells or (2) the presence of immunogenic necrotic cells. This strategy, however, is ineffective when the battlefield is plain or uphill, or when the tumor itself promotes a "need to repair" response due to chemotherapy-induced apoptosis or other unknown factors. Thus, when faced with this scenario, an approach to ensure that the immune system senses the cancer as danger in order to promote a "need to fight" over a "need to repair" response is essential. Consequently, the "push the accelerator" component of the universal magic bullet is still lacking. This action may possibly be achieved by educating APCs (DCs and macrophages) to sense every cancer as "dangerous", ensuring an anti-tumor immune response in all cases. Moreover, understanding that certain immune cells (e.g. macrophages) can shift the immune response in one direction over another, combination therapies may be necessary to transiently eliminate other immune cells. Ultimately, the goal is to unmask those factors that promote a "need to repair" response while at the same time enhancing those factors that are sensed as a danger signal by the immune system. Only then will immunotherapy truly become the magic bullet for cancer treatment.

\section{Abbreviations}

DC: dendritic cells; APC: antigen presenting cells; IL: interleukin; NK: natural killer; $T_{\text {reg: }}$ T-regulatory cell; CTLA-4: cytotoxic T-Lymphocyte antigen-4; PD-1: programmed cell death protein 1; PD-L1: programmed death-ligand 1; PBMC: peripheral blood mononuclear cells; IFN: interferon; TNF: tumor necrosis factor; GM-CSF: granulocyte-macrophage colony-stimulating factor; TAM: tumor-associated macrophage; TAN: tumor-associated neutrophil.

\section{Authors' contributions}

GD prepared, designed and wrote the manuscript. HLM and BSJ assisted in the manuscript design and edited the manuscript. All authors read and approved the final manuscript.

\section{Author details}

${ }^{1}$ Department of Biochemistry, School of Medicine, Autónoma University of Madrid, Calle del Arzobispo Morcillo 4, 28029 Madrid, Spain. ${ }^{2}$ Department of Biochemistry and Molecular Biology, Tulane University School of Medicine, 1430 Tulane Ave, \#8543, New Orleans, LA 70112, USA. ${ }^{3}$ Department of Cancer Biology, Instituto de Investigaciones Biomédicas "Alberto Sols" CSIC-UAM, Madrid, Spain. ${ }^{4}$ Enfermedades Crónicas y Cáncer Area, Instituto Ramón y Cajal de Investigación Sanitaria (IRYCIS), Madrid, Spain.

\section{Acknowledgements}

The authors would like to thank their respective laboratories and clinical collaborators for helpful discussions.

\section{Competing interests}

The authors declare that they have no competing interests.

\section{Funding}

This work was supported by NIH R00 CA154605 and Louisiana Board of Regents LEQSF(2016-17)-RD-C-14 (H.L.M.), a Rámon y Cajal Merit Award from the Ministerio de Economía y Competitividad, Spain (B.S.Jr) and a Clinic and Laboratory Integration Program (CLIP) grant from the Cancer Research Institute, NY (B.S.Jr).

Received: 24 October 2016 Accepted: 7 December 2016 Published online: 03 January 2017 


\section{References}

1. DeVita VT Jr, Chu E (2008) A history of cancer chemotherapy. Cancer Res 68:8643-8653

2. Liang XJ, Chen C, Zhao Y et al (2010) Circumventing tumor resistance to chemotherapy by nanotechnology. Methods Mol Biol 596:467-488

3. Luqmani YA (2005) Mechanisms of drug resistance in cancer chemotherapy. Med Princ Pract 14(Suppl 1):35-48

4. Attarwala $\mathrm{H}$ (2010) Role of antibodies in cancer targeting. J Nat Sci Biol Med 1:53-56

5. Reslan L, Dalle S, Dumontet C (2009) Understanding and circumventing resistance to anticancer monoclonal antibodies. MAbs 1:222-229

6. Burnet F, Fenner F (1949) The production of antibodies, 2nd edn. Macmillan, Melbourne

7. Coussens LM, Zitvogel L, Palucka AK (2013) Neutralizing tumor-promoting chronic inflammation: a magic bullet? Science 339:286-291

8. McCarthy EF (2006) The toxins of William B. Coley and the treatment of bone and soft-tissue sarcomas. lowa Orthop J 26:154-158

9. Steen S, Stephenson G (2008) Current treatment of soft tissue sarcoma. Proc (Bayl Univ Med Cent) 21:392-396

10. Alloatti A, Kotsias F, Magalhaes JG et al (2016) Dendritic cell maturation and cross-presentation: timing matters! Immunol Rev 272:97-108

11. Morelli AE, O'Connell PJ, Khanna A et al (2000) Preferential induction of Th1 responses by functionally mature hepatic (CD8alpha- and CD8alpha+) dendritic cells: association with conversion from liver transplant tolerance to acute rejection. Transplantation 69:2647-2657

12. Agrawal S, Agrawal A, Said HM (2016) Biotin deficiency enhances the inflammatory response of human dendritic cells. Am J Physiol Cell Physiol 00141:02016

13. Romagnani S (2000) T-cell subsets (Th1 versus Th2). Ann Allergy Asthma Immunol 85:9-18 (quiz 18, 21)

14. Chen J, Zurawski G, Zurawski S et al (2015) A novel vaccine for mantle cell lymphoma based on targeting cyclin D1 to dendritic cells via CD40. J Hematol Oncol 8:35

15. Liston A (2011) Immunological tolerance 50 years after the Burnet Nobel Prize. Immunol Cell Biol 89:14-15

16. Billingham RE, Brent L, Medawar PB (1953) Actively acquired tolerance of foreign cells. Nature 172:603-606

17. Lederberg J (1959) Genes and antibodies. Science 129:1649-1653

18. Nossal GJ, Pike BL (1978) Mechanisms of clonal abortion tolerogenesis. I. Response of immature hapten-specific B lymphocytes. J Exp Med 148:1161-1170

19. Marrack P, Kappler J (1990) T cell tolerance. Semin Immunol 2:45-49

20. Goodnow CC, Adelstein S, Basten A (1990) The need for central and peripheral tolerance in the B cell repertoire. Science 248:1373-1379

21. Le Douarin N, Corbel C, Bandeira A et al (1996) Evidence for a thymusdependent form of tolerance that is not based on elimination or anergy of reactive T cells. Immunol Rev 149:35-53

22. Foley EJ (1953) Antigenic properties of methylcholanthrene-induced tumors in mice of the strain of origin. Cancer Res 13:835-837

23. Nathrath WB (1978) Organ and tumour antigens in malignant disease: a review. J R Soc Med 71:755-761

24. Hargraves MM (1969) Discovery of the LE cell and its morphology. Mayo Clin Proc 44:579-599

25. DeLeo AB, Jay G, Appella E et al (1979) Detection of a transformationrelated antigen in chemically induced sarcomas and other transformed cells of the mouse. Proc Natl Acad Sci USA 76:2420-2424

26. Boon T, Cerottini JC, Van den Eynde B et al (1994) Tumor antigens recognized by T lymphocytes. Annu Rev Immunol 12:337-365

27. Salmi M, Hellman J, Jalkanen S (1998) The role of two distinct endothelial molecules, vascular adhesion protein-1 and peripheral lymph node addressin, in the binding of lymphocyte subsets to human lymph nodes. J Immunol 160:5629-5636

28. Steinman RM (2007) Lasker basic medical research award. Dendritic cells: versatile controllers of the immune system. Nat Med 13:1155-1159

29. Bronte V, Pittet MJ (2013) The spleen in local and systemic regulation of immunity. Immunity 39:806-818

30. Vasilevko V, Ghochikyan A, Holterman MJ et al (2002) CD80 (B7-1) and CD86 (B7-2) are functionally equivalent in the initiation and maintenance of CD4+ T-cell proliferation after activation with suboptimal doses of PHA. DNA Cell Biol 21:137-149
31. Sprent J (1994) T and B memory cells. Cell 76:315-322

32. Schietinger A, Greenberg PD (2014) Tolerance and exhaustion: defining mechanisms of T cell dysfunction. Trends Immunol 35:51-60

33. Sprent J, Kishimoto $H$ (2001) The thymus and central tolerance. Philos Trans R Soc Lond B Biol Sci 356:609-616

34. Schwartz RH (1990) A cell culture model for T lymphocyte clonal anergy. Science 248:1349-1356

35. Mueller DL, Jenkins MK, Schwartz RH (1989) Clonal expansion versus functional clonal inactivation: a costimulatory signalling pathway determines the outcome of T cell antigen receptor occupancy. Annu Rev Immunol 7:445-480

36. Brunet JF, Denizot F, Luciani MF et al (1987) A new member of the immunoglobulin superfamily-CTLA-4. Nature 328:267-270

37. van der Merwe PA, Bodian DL, Daenke S et al (1997) CD80 (B7-1) binds both CD28 and CTLA-4 with a low affinity and very fast kinetics. J Exp Med 185:393-403

38. Lipson EJ, Drake CG (2011) Ipilimumab: an anti-CTLA-4 antibody for metastatic melanoma. Clin Cancer Res 17:6958-6962

39. Waterhouse P, Penninger JM, Timms E et al (1995) Lymphoproliferative disorders with early lethality in mice deficient in Ctla-4. Science 270:985-988

40. Leach DR, Krummel MF, Allison JP (1996) Enhancement of antitumor immunity by CTLA-4 blockade. Science 271:1734-1736

41. van Elsas A, Hurwitz AA, Allison JP (1999) Combination immunotherapy of B16 melanoma using anti-cytotoxic T lymphocyte-associated antigen 4 (CTLA-4) and granulocyte/macrophage colony-stimulating factor (GM-CSF)-producing vaccines induces rejection of subcutaneous and metastatic tumors accompanied by autoimmune depigmentation. J Exp Med 190:355-366

42. van Elsas A, Sutmuller RP, Hurwitz AA et al (2001) Elucidating the autoimmune and antitumor effector mechanisms of a treatment based on cytotoxic T lymphocyte antigen-4 blockade in combination with a B16 melanoma vaccine: comparison of prophylaxis and therapy. J Exp Med 194:481-489

43. Lute KD, May KF Jr, Lu P et al (2005) Human CTLA4 knock-in mice unravel the quantitative link between tumor immunity and autoimmunity induced by anti-CTLA-4 antibodies. Blood 106:3127-3133

44. Keler T, Halk E, Vitale L et al (2003) Activity and safety of CTLA-4 blockade combined with vaccines in cynomolgus macaques. J Immunol 171:6251-6259

45. Ghiotto M, Gauthier L, Serriari N et al (2010) PD-L1 and PD-L2 differ in their molecular mechanisms of interaction with PD-1. Int Immunol 22:651-660

46. Latchman Y, Wood CR, Chernova T et al (2001) PD-L2 is a second ligand for PD-1 and inhibits T cell activation. Nat Immunol 2:261-268

47. Dong H, Zhu G, Tamada K et al (1999) B7-H1, a third member of the B7 family, co-stimulates T-cell proliferation and interleukin-10 secretion. Nat Med 5:1365-1369

48. Ishida Y, Agata Y, Shibahara K et al (1992) Induced expression of PD-1, a novel member of the immunoglobulin gene superfamily, upon programmed cell death. EMBO J 11:3887-3895

49. Agata $Y$, Kawasaki A, Nishimura $\mathrm{H}$ et al (1996) Expression of the PD-1 antigen on the surface of stimulated mouse $T$ and B lymphocytes. Int Immunol 8:765-772

50. Francisco LM, Salinas VH, Brown KE et al (2009) PD-L1 regulates the development, maintenance, and function of induced regulatory $T$ cells. J Exp Med 206:3015-3029

51. Jin HT, Ahmed R, Okazaki T (2011) Role of PD-1 in regulating T-cell immunity. Curr Top Microbiol Immunol 350:17-37

52. Sheppard KA, Fitz LJ, Lee JM et al (2004) PD-1 inhibits T-cell receptor induced phosphorylation of the ZAP70/CD3zeta signalosome and downstream signaling to PKCtheta. FEBS Lett 574:37-41

53. Barber DL, Wherry EJ, Masopust D et al (2006) Restoring function in exhausted CD8 T cells during chronic viral infection. Nature 439:682-687

54. Dong H, Strome SE, Salomao DR et al (2002) Tumor-associated B7-H1 promotes T-cell apoptosis: a potential mechanism of immune evasion. Nat Med 8:793-800

55. Niezgoda A, Niezgoda P, Czajkowski R (2015) Novel approaches to treatment of advanced melanoma: a review on targeted therapy and immunotherapy. Biomed Res Int 2015:851387 
56. Topalian SL, Sznol M, McDermott DF et al (2014) Survival, durable tumor remission, and long-term safety in patients with advanced melanoma receiving nivolumab. J Clin Oncol 32:1020-1030

57. Keating GM (2016) Nivolumab: a review in advanced nonsquamous non-small cell lung cancer. Drugs 76:969-978

58. Larkin J, Chiarion-Sileni V, Gonzalez R et al (2015) Combined nivolumab and ipilimumab or monotherapy in untreated melanoma. N Engl J Med 373:23-34

59. Kantoff PW, Higano CS, Shore ND et al (2010) Sipuleucel-T immunotherapy for castration-resistant prostate cancer. N Engl J Med 363:411-422

60. Lutz ER, Wu AA, Bigelow E et al (2014) Immunotherapy converts nonimmunogenic pancreatic tumors into immunogenic foci of immune regulation. Cancer Immunol Res 2:616-631

61. Nummer D, Suri-Payer E, Schmitz-Winnenthal H et al (2007) Role of tumor endothelium in CD4+ CD25+ regulatory T cell infiltration of human pancreatic carcinoma. J Natl Cancer Inst 99:1188-1199

62. Sainz B Jr, Carron E, Vallespinos M et al (2016) Cancer stem cells and macrophages: implications in tumor biology and therapeutic strategies. Mediat Inflamm 2016:9012369

63. Bui JD, Uppaluri R, Hsieh CS et al (2006) Comparative analysis of regulatory and effector $T$ cells in progressively growing versus rejecting tumors of similar origins. Cancer Res 66:7301-7309

64. Diller ML, Kudchadkar RR, Delman KA et al (2016) Balancing inflammation: the link between Th17 and regulatory T cells. Mediat Inflamm 2016:6309219

65. Shaw TJ, Martin P (2016) Wound repair: a showcase for cell plasticity and migration. Curr Opin Cell Biol 42:29-37

66. DuPage M, Bluestone JA (2016) Harnessing the plasticity of CD4(+) T cells to treat immune-mediated disease. Nat Rev Immunol 16:149-163

67. Zhu J, Yamane H, Paul WE (2010) Differentiation of effector CD4 T cell populations. Annu Rev Immunol 28:445-489

68. Palucka K, Banchereau J (2012) Cancer immunotherapy via dendritic cells. Nat Rev Cancer 12:265-277

69. Martinez FO, Gordon S (2014) The M1 and M2 paradigm of macrophage activation: time for reassessment. F1000Prime Rep 6:13

70. Mantovani A, Cassatella MA, Costantini C et al (2011) Neutrophils in the activation and regulation of innate and adaptive immunity. Nat Rev Immunol 11:519-531

71. Steinman RM (2007) Dendritic cells: understanding immunogenicity. Eur J Immunol 37(Suppl 1):S53-S60

72. Fujii S, Liu K, Smith C et al (2004) The linkage of innate to adaptive immunity via maturing dendritic cells in vivo requires CD40 ligation in addition to antigen presentation and CD80/86 costimulation. J Exp Med 199:1607-1618

73. Maldonado RA, von Andrian UH (2010) How tolerogenic dendritic cells induce regulatory T cells. Adv Immunol 108:111-165

74. Lee Jl, Ganster RW, Geller DA et al (1999) Cyclosporine A inhibits the expression of costimulatory molecules on in vitro-generated dendritic cells: association with reduced nuclear translocation of nuclear factor kappa B. Transplantation 68:1255-1263

75. Penna G, Adorini L (2000) 1 alpha, 25-dihydroxyvitamin D3 inhibits differentiation, maturation, activation, and survival of dendritic cells leading to impaired alloreactive T cell activation. J Immunol 164:2405-2411

76. Gordon S, Martinez FO (2010) Alternative activation of macrophages: mechanism and functions. Immunity 32:593-604

77. Kidd JF, Pilkington MF, Schell MJ et al (2002) Paclitaxel affects cytosolic calcium signals by opening the mitochondrial permeability transition pore. J Biol Chem 277:6504-6510

78. Sica A, Schioppa T, Mantovani A et al (2006) Tumour-associated macrophages are a distinct $\mathrm{M} 2$ polarised population promoting tumour progression: potential targets of anti-cancer therapy. Eur J Cancer 42:717-727

79. Rath M, Muller I, Kropf P et al (2014) Metabolism via arginase or nitric oxide synthase: two competing arginine pathways in macrophages. Front Immunol 5:532

80. Golpon HA, Fadok VA, Taraseviciene-Stewart L et al (2004) Life after corpse engulfment: phagocytosis of apoptotic cells leads to VEGF secretion and cell growth. FASEB J 18:1716-1718

81. Zhao Y, Zhou FL, Li WP et al (2016) Slit2Robo1 signaling promotes the adhesion, invasion and migration of tongue carcinoma cells via upregulating matrix metalloproteinases 2 and 9, and downregulating Ecadherin. Mol Med Rep 14:1901-1906
82. Jakubowska K, Pryczynicz A, Januszewska J et al (2016) Expressions of matrix metalloproteinases 2,7, and 9 in carcinogenesis of pancreatic ductal adenocarcinoma. Dis Markers 2016:9895721

83. Huber S, Hoffmann R, Muskens F et al (2010) Alternatively activated macrophages inhibit T-cell proliferation by Stat6-dependent expression of PD-L2. Blood 116:3311-3320

84. Zhang L, Conejo-Garcia JR, Katsaros D et al (2003) Intratumoral T cells, recurrence, and survival in epithelial ovarian cancer. $N$ Engl J Med 348:203-213

85. Geissler K, Fornara P, Lautenschlager C et al (2015) Immune signature of tumor infiltrating immune cells in renal cancer. Oncoimmunology 4:e985082

86. Sato E, Olson SH, Ahn J et al (2005) Intraepithelial CD8+ tumor-infiltrating lymphocytes and a high CD8+/regulatory $T$ cell ratio are associated with favorable prognosis in ovarian cancer. Proc Natl Acad Sci USA 102:18538-18543

87. Li X, Kostareli E, Suffner J et al (2010) Efficient Treg depletion induces T-cell infiltration and rejection of large tumors. Eur J Immunol 40:3325-3335

88. Bonavita E, Galdiero MR, Jaillon S et al (2015) Phagocytes as corrupted policemen in cancer-related inflammation. Adv Cancer Res 128:141-171

89. Nathan C (2002) Points of control in inflammation. Nature 420:846-852

90. Beaman KD, Jaiswal MK, Katara GK et al (2016) Pregnancy is a model for tumors, not transplantation. Am J Reprod Immunol 76:3-7

91. Vakkila J, Lotze MT (2004) Inflammation and necrosis promote tumour growth. Nat Rev Immunol 4:641-648

92. Harris CC (1976) The carcinogenicity of anticancer drugs: a hazard in man. Cancer 37:1014-1023

93. Iyer SS, Pulskens WP, Sadler JJ et al (2009) Necrotic cells trigger a sterile inflammatory response through the Nlrp3 inflammasome. Proc Natl Acad Sci USA 106:20388-20393

94. Thompson CB (1995) Apoptosis in the pathogenesis and treatment of disease. Science 267:1456-1462

95. Casares N, Pequignot MO, Tesniere A et al (2005) Caspase-dependent immunogenicity of doxorubicin-induced tumor cell death. J Exp Med 202:1691-1701

96. Okada M, Adachi S, Imai T et al (2004) A novel mechanism for imatinib mesylate-induced cell death of BCR-ABL-positive human leukemic cells: caspase-independent, necrosis-like programmed cell death mediated by serine protease activity. Blood 103:2299-2307

97. Campisi L, Cummings RJ, Blander JM (2014) Death-defining immune responses after apoptosis. Am J Transplant 14:1488-1498

98. Ferguson TA, Herndon J, Elzey B et al (2002) Uptake of apoptotic antigen-coupled cells by lymphoid dendritic cells and cross-priming of CD8(+) T cells produce active immune unresponsiveness. J Immunol 168:5589-5595

99. Griffith TS, Kazama H, VanOosten RL et al (2007) Apoptotic cells induce tolerance by generating helpless CD8+ T cells that produce TRAIL. J Immunol 178:2679-2687

100. Tomimori Y, Ikawa Y, Oyaizu N (2000) Ultraviolet-irradiated apoptotic lymphocytes produce interleukin-10 by themselves. Immunol Lett 71:49-54

101. Weigert A, Tzieply N, von Knethen A et al (2007) Tumor cell apoptosis polarizes macrophages role of sphingosine-1-phosphate. Mol Biol Cell 18:3810-3819

102. Williams CA, Harry RA, McLeod JD (2008) Apoptotic cells induce dendritic cell-mediated suppression via interferon-gamma-induced IDO. Immunology 124:89-101

103. Nakahashi-Oda C, Udayanga KG, Nakamura Y et al (2016) Apoptotic epithelial cells control the abundance of Treg cells at barrier surfaces. Nat Immunol 17:441-450

104. Kleinclauss F, Perruche S, Masson E et al (2006) Intravenous apoptotic spleen cell infusion induces a TGF-beta-dependent regulatory T-cell expansion. Cell Death Differ 13:41-52

105. Wu C, Zhang Y, Jiang Y et al (2013) Apoptotic cell administration enhances pancreatic islet engraftment by induction of regulatory $T$ cells and tolerogenic dendritic cells. Cell Mol Immunol 10:393-402 